\title{
Voz do professor: uma revisão de 15 anos de contribuição fonoaudiológica
}

\author{
Teachers' voice: a review of 15 years of SLP contribution
}

\author{
Maria Lúcia Suzigan Dragone ${ }^{1}$, Léslie Piccolotto Ferreira ${ }^{2}$, Susana Pimentel Pinto Giannini \\ Marcia Simões-Zenari ${ }^{4}$, Vanessa Pedrosa Vieira ${ }^{5}$, Mara Behlau $^{6}$
}

\begin{abstract}
RESUMO
O objetivo deste artigo foi realizar uma revisão das publicações brasileiras referentes à voz do professor, produzidas por fonoaudiólogos, no período de 1994 a 2008. O estudo transversal, de natureza bibliográfica, foi realizado em três etapas. A primeira constou de levantamento em diferentes fontes; a segunda compreendeu a busca dos resumos de todos os trabalhos relacionados; na terceira, foi realizada leitura do material para classificação em quatro categorias: avaliação dos indivíduos; avaliação dos efeitos de intervenções; descrição de intervenções; e estudos bibliográficos/teóricos/documentais. Foram contabilizadas 500 publicações, sendo 415 (83\%) referentes às categorias de avaliações e, dentre essas, 357 (86\%) com objetivo de avaliação dos participantes e/ou das condições de trabalho e apenas 58 (14\%) com preocupação de avaliar os efeitos de programas/intervenções. Quanto às dimensões de análise das avaliações, que em 202 estudos (48,7\%) foram mais de uma, a perspectiva dos próprios professores foi a mais utilizada $(52,5 \%$ das análises), seguida por análises perceptivo-auditivas realizadas por fonoaudiólogos (15,2\%), e avaliação do ambiente e da organização do trabalho $(14,9 \%)$. Na categoria denominada descritivos de intervenção foram encontrados $31(6,2 \%)$ trabalhos e na categoria bibliográfico/teórico/documental, 54 (10,8\%). A revisão realizada confirma que a perspectiva da avaliação vocal tem sido privilegiada nos estudos brasileiros. Avaliações de efeito de intervenções, mais recentes e ainda pouco representativas, indicam uma tendência de mudança de foco que pode auxiliar a compreensão da complexa realidade do uso da voz na docência, nortear futuras ações fonoaudiológicas e inspirar estudos sobre intervenção fonoaudiológica com esses indivíduos.
\end{abstract}

Descritores: Voz; Docentes; Pesquisas; Literatura de revisão como assunto; Distúrbios da voz

Trabalho realizado em colaboração nas instituições: UNIARA, PUC-SP, USP-SP, CEV-SP e UNIFESP.

(1) Doutora, Professora do Curso de Fonoaudiologia e de Pedagogia do Centro Universitário de Araraquara - UNIARA - Araraquara (SP), Brasil.

(2) Doutora, Professora Titular da Faculdade de Fonoaudiologia e do Programa de Estudos Pós-Graduados em Fonoaudiologia da Pontifícia Universidade Católica de São Paulo - PUC-SP - São Paulo (SP), Brasil; Professora do Curso de Especialização em Fonoaudiologia - Voz da Pontifícia Universidade Católica de São Paulo - PUC-SP - São Paulo (SP), Brasil.

(3) Doutora, Professora do Curso de Especialização em Fonoaudiologia - Voz da Pontifícia Universidade Católica de São Paulo - PUC-SP - São Paulo (SP), Brasil; Fonoaudióloga do Hospital do Servidor Público Municipal de São Paulo - HSPM e da Divisão de Reabilitação dos Distúrbios da Comunicação da Pontifícia Universidade Católica de São Paulo - PUC-SP - São Paulo (SP), Brasil.

(4) Doutora, Fonoaudióloga do Departamento de Fisioterapia, Fonoaudiologia e Terapia Ocupacional da Faculdade de Medicina da Universidade de São Paulo - USP - São Paulo (SP), Brasil.

(5) Mestre, Consultora de Pesquisas do Centro Cochrane do Brasil - UNIFESP - São Paulo (SP), Brasil; Professora do Curso de Especialização em Voz do Centro de Estudos da Voz - CEV - São Paulo (SP), Brasil.

(6) Doutora, Professora do Programa de Pós-Graduação em Distúrbios da Comunicação Humana da Universidade Federal de São Paulo - UNIFESP

- São Paulo (SP), Brasil; Professora do Curso de Especialização em Voz do Centro de Estudos da Voz - CEV - SãoPaulo (SP), Brasil.

Endereço para correspondência: Maria Lucia Suzigan Dragone. Av. Prof. Augusto Cezar, 902,Araraquara (SP), Brasil,CEP: 14801-240. E-mail: mldragone@uol.com.br

Recebido em: 15/3/2010; Aceito em: 4/5/2010

\section{INTRODUÇÃO}

Estudos de revisão têm reconhecida importância na área da saúde e, embora ainda sejam pouco numerosos na Fonoaudiologia, vêm aumentando na última década. Neles, há ênfase para a necessidade de reflexões em torno das temáticas utilizadas e identificação de novos rumos a partir das análises realizadas ${ }^{(1-3)}$.

A presença de estudos abordando diagnóstico/avaliação compõe a maioria das publicações $(537=68,2 \%)$ na área dos Distúrbios da Comunicação em periódicos nacionais da Fonoaudiologia ${ }^{(4)}$ no período de 2000 a 2005 . A temática voz é privilegiada somente em 8,9\% (71) dos estudos e, destes, 41 (65\%) envolvem diagnóstico/avaliação, nove $(14,3 \%)$ relatos de ações em assessoria/promoção, seguidos por seis relatos de atuação em fonoterapia individual (9,5\%), e outros enfoques como triagem e combinados de diagnóstico/avaliação/terapia ( $2=3,2 \%$ cada um). A mesma tendência em privilegiar estudos sobre avaliações $(59=44,7 \%)$ aparece em estudo subsequente somente com publicações em voz ${ }^{(5)}$, nas quais o foco está em voz profissional $(43=32,6 \%)$, descritivos de processo terapêutico $(17=12,9 \%)$ e pouca contribuição sobre promoção de saúde/prevenção de alterações vocais $(6=4,5 \%)$. No entanto, em recente revisão sistemática de publicações em voz e laringe em duas revistas científicas nacionais e duas internacionais ${ }^{(6)}$, 
observa-se menor ocorrência de estudos com objetivo principal de avaliação como diagnóstico primário $(101=27 \%)$ mantendo-se a baixa ocorrência de estudos abordando prevenção $(4=1,1 \%)$.

As razões para a investigação voltada mais para avaliação (diagnóstico/ocorrência), o aumento da quantidade de publicações, e os tipos de divulgação com maior ocorrência em congressos são históricas, visto os achados em revisão de estudos em saúde em geral $^{(7)}$ : no período de 1973 a 1992, entre as publicações produzidas em cinco países da América Latina, $53,4 \%$ dos estudos têm foco na área clínica e somente 2,7\% em saúde pública; nota-se, entre estudos brasileiros da área da saúde, acentuado aumento de publicações no período de 1983 a 1993 relacionado à produção de programas de pósgraduação no Brasil, mantendo-se um ritmo de crescimento de $20 \%$ depois deste período; entre 1990 a 1994 os temas mais presentes das teses brasileiras na saúde são epidemiologia de doenças e promoção de saúde, com divulgação em congressos nacionais e internacionais $(58,1 \%)$, periódicos nacionais $(35,4 \%)$, livros $(10,5 \%)$ e capítulos de livros $(8,2 \%)$. As recomendações de atenção especial para que os estudos sejam, no futuro, mais aprofundados, com melhor formatação, construção de hipóteses e utilização de métricas científicas de forma a contribuírem para análise em Saúde Coletiva estão também presentes nesta revisão $0^{(7)}$.

As publicações fonoaudiológicas na área de voz profissional $^{(8-11)}$ têm sido, nos últimos anos, um dos focos das atividades científicas do Departamento de Voz da Sociedade Brasileira de Fonoaudiologia. A primeira publicação ${ }^{(12)}$ sobre voz do professor é da década de 80, sem registro de outra até 1993; desta data até os dias atuais, mantém-se um ritmo crescente de publicações, sendo o professor o profissional de voz mais pesquisado.

A produção expressiva em voz do professor pode ser explicada pelo grande número de docentes em nosso país, assim como em outros países, que comumente procuram as clínicas médicas devido ao problema de $\mathrm{voz}^{(13)}$, tornando-se sujeito de pesquisa de fácil acesso. Além, disso,há indicação consistente de que esta categoria profissional apresenta maior prevalência de distúrbios vocais quando comparada à população em geral ${ }^{(14,15)}$.

Nas teses abordando as condições de trabalho e saúde de professores da educação básica no Brasil ${ }^{(16)}$ percebe-se um aumento de interesse em pesquisar a voz do professor na última década. A maioria $(14=93,3 \%)$ das 15 dissertações e teses selecionadas sobre o assunto foi apresentada após o ano 2000. Há, neste caso,tendência em correlacionar causaefeito na busca de fatores de risco, apesar de se perceber interesse em analisar outras dimensões do problema (sociais, históricas do trabalho e do processo de produção da saúde/ doença) distantes da sala de aula. Há utilização de protocolos internacionais de auto-avaliação traduzidos e validados para o Brasil, investigando qualidade de vida e as relações entre estresse e mal estar docente.

As alterações vocais em professores decorrentes do trabalho são, também, analisadas em uma revisão de artigos de periódicos indexados na base SciELO, publicados no período de 1998 a $2008^{(17)}$. Das 15 fontes identificadas que contempla- ram as alterações de voz em professores, são mais frequentes os estudos de delineamento transversal $(33,38 \%)$, com professores de ambos os sexos em $60 \%$ deles e,prioritariamente, do ensino fundamental $(53,33 \%)$.Os temas principais são a identificação de problemas relacionados à voz,conhecimento e cuidados vocais, comportamentos inapropriados e promoção de saúde vocal.

Essa variabilidade na terminologia das temáticas, conforme observado nos estudos sobre voz em geral ${ }^{(18)}$, inclui, além de terminologia vaga, linhas de pesquisa muitas vezes não adequadas às perguntas do estudo, interpretações pouco cuidadosas de medidas comportamentais e psicológicas, e necessidade de realização de intervenções cada vez mais mensuráveis ${ }^{(6,18)}$ para validar os procedimentos de promoção e cuidados em saúde vocal.

Modificações de enfoques dos inúmeros estudos da Fonoaudiologia brasileira sobre voz do professor aconteceram no decorrer do tempo. Estudos abordando a ocorrência de distúrbios de voz entre os professores e de mapeamento de fatores intercorrentes são os de maior ocorrência $(155=50,5 \%)$ entre 307 publicações ${ }^{(19)}$ científicas fonoaudiológicas sobre voz professor até 2005, excluindo-se aquelas repetidas em fontes diversas (periódicos, teses, livros, capítulos de livros, anais de eventos científicos, trabalho de conclusão de cursos). A partir do ano 2000, entre as investigações sobre voz no trabalho, há aumento de interesse por estudos do ambiente escolar e condições de trabalho do professor (até 1999 com 5=22,7\%; depois de 2000 com $17=77,3 \%$ dos 22 com este foco) e de estudos sobre a voz como recurso didático do professor (até 1999 com $1=5,9 \%$, depois de 2000 com $16=94,1 \%$ dos 17 com este foco). Os estudos que relacionam voz do professor e qualidade de vida têm maior ocorrência entre 2002 e 2004 (10=83,3\% dos 12 estudos deste foco), dado associado diretamente à tradução e validação no Brasil de protocolos internacionais de auto-avaliação; mas, estudos que avaliam os resultados de intervenções ainda são escassos $(7=2,3 \%)$ entre os analisados. O levantamento em questão ${ }^{(19)}$ despertou o interesse em mapear de maneira mais aprofundada, e ampliada, estes dados reveladores do percurso das pesquisas sobre voz do professor e norteadores para futuros focos de interesse.

O objetivo do presente estudo é analisar 15 anos (1994 a 2008) de contribuição das publicações fonoaudiológicas brasileiras para a compreensão da voz do professor e identificar suas características quanto ao tipo de publicações e enfoque dos estudos.

\section{REVISÃO DA LITERATURA}

A proposta partiu do delineamento de um estudo descritivo, exploratório e transversal, de natureza bibliográfica, que aborda a temática da voz do professor, na Fonoaudiologia, por um período de 15 anos - 1994 a 2008.

A revisão de literatura foi feita em três etapas: levantamento do material, classificação e análise de conteúdo.

A primeira etapa, de levantamento dos trabalhos realizados sobre o assunto no período citado, foi feita considerando-se as seguintes bases de pesquisa: portal da Biblioteca Regional de Medicina (BIREME), sítio do Scientific Electronic Library On 
Line (SciELO), Banco de Dissertações e Teses organizado pela Coordenação de Aperfeiçoamento do Ensino Superior (Portal CAPES), bibliotecas de instituições de ensino (virtuais e de consulta física), nas quais foram consultados livros, capítulos de livros, revistas científicas, anais de congressos, trabalhos de conclusão de curso ou iniciação científica,monografias de especialização, dissertações e teses, produções digitalizadas (CD), além de solicitação por contato direto com os autores dos trabalhos científicos da área. Foi inicialmente feita uma busca a partir do título do trabalho e, em seguida, coleta dos respectivos resumos.

As palavras-chave usadas de forma isolada ou em cruzamento foram: voz, voz profissional, voz do professor, distúrbio da voz, disfonia, professor(es), ensino, docência e trabalho.

Nessa primeira etapa, foram levantados 636 títulos, com pelo menos um fonoaudiólogo como autor do trabalho. Foram excluídos 136 títulos $(21,4 \%)$, pelos seguintes critérios: publicação fora do período estipulado $(5 ; 0,8 \%)$, textos não científicos, resumos não encontrados $(27 ; 4,2 \%)$ e mesma publicação em diversos meios (104; 16,4\%). Quanto a este último critério, principalmente no que se refere à produção de dissertações e teses, verificou-se uma tendência dos autores em procurar a máxima visibilidade de seu estudo, o que implica publicações múltiplas (anais de jornadas e congressos, revistas e capítulos de livros). Manteve-se apenas a entrada principal favorecendo-se em primeiro lugar, a publicação de artigos científicos, por ser a mais importante divulgação. Os 500 resumos remanescentes foram organizados para classificação.

A segunda etapa compreendeu a classificação e análise dos resumos levantados, feita em duas dimensões: tipo de publicação e enfoque do estudo, analisadas também de acordo com o ano da publicação (Tabela 1). O tipo de publicação foi classificado em nove categorias: resumo em anais de eventos científicos (338; 67,6\%); artigo $(69 ; 13,8 \%)$; tese ou dissertação de mestrado ( $34 ; 6,8 \%$ ); monografia de especialização ( 25 ; $5 \%)$; capítulo de livro $(20 ; 4 \%)$; doutorado $(7 ; 1,4 \%)$; monografias de iniciação científica $(3 ; 6 \%)$, trabalho de conclusão de curso ( $3 ; 6 \%)$, e livro ( $1 ; 0,2 \%)$. O enfoque dos 500 estudos foi classificado em quatro categorias (Tabela 2): avaliação do professor $(353 ; 70,6 \%)$, estudos bibliográficos, teóricos ou documentais $(54 ; 10,8 \%)$, avaliação dos efeitos de intervenções $(46 ; 9,2 \%)$, descrição de intervenções $(31 ; 6,2 \%)$, e, uma adicional de estudos que enfocaram mais de uma categoria $(16 ; 3,2 \%)$. As categorias avaliação do professor e avaliação do efeito de intervenções foram ainda sub categorizadas de acordo com a perspectiva de análise (Tabela 3): auto-referida (relato/opinião do próprio professor, $366 ; 52,3 \%$ ), avaliação perceptivo-auditiva $(107 ; 15,3 \%)$, ambiental/organizacional do trabalho (105; 15\%), avaliação comportamental $(42 ; 6 \%)$, análise acústica $(34 ; 4,8 \%)$, avaliação médica $(33 ; 4,7 \%)$, avaliação e não especificados (12; 1,7\%). Ressalta-se ainda que, dos 415 estudos de avaliação, $213(51,3 \%)$ analisaram apenas uma dimensão, 139 duas (34,5\%), 51 (12,3\%) e 12 estudos $(2,9 \%)$ analisaram mais de três dimensões. Entre os 151 estudos que referem, nos resumos, dados sobre alteração vocal (Tabela 4), observou-se que estes foram obtidos por duas vias principais: auto-avaliação do professor $(115 ; 76,2 \%)$ e avaliação fonoaudiológica (36; 23,8\%); estes dados foram obtidos principalmente com questionários respondidos pelos professores $(319 ; 66,6 \%)$, seguidos por amostras de voz registradas em áudio $(91 ; 19 \%)$ e outros instrumentos de coleta de dados como entrevistas, grupo focal, observação in loco, registros de imagem, imagem de laringe, prontuários, com menos de $10 \%$ de ocorrência cada um (Tabela 4).

Há evidente aumento das publicações a partir de 1999, quando a ocorrência aumentou em mais de 60\% (1994 a 1998

Tabela 1. Tipo de publicação

\begin{tabular}{|c|c|c|c|c|c|c|c|c|c|c|c|c|c|c|c|c|c|c|c|c|c|c|}
\hline \multirow[t]{2}{*}{ Ano } & \multicolumn{2}{|c|}{$\begin{array}{c}\text { Resumo em } \\
\text { anais }\end{array}$} & \multicolumn{2}{|c|}{ Artigo } & \multicolumn{2}{|c|}{$\begin{array}{l}\text { Tese ou dis- } \\
\text { sertação de } \\
\text { mestrado }\end{array}$} & \multicolumn{2}{|c|}{$\begin{array}{c}\text { Monografia } \\
\text { especializa- } \\
\text { ção }\end{array}$} & \multicolumn{2}{|c|}{$\begin{array}{l}\text { Capítulo } \\
\text { de livro }\end{array}$} & \multicolumn{2}{|c|}{ Doutorado } & \multicolumn{2}{|c|}{$\begin{array}{c}\text { Monografias } \\
\text { de iniciação } \\
\text { científica }\end{array}$} & \multicolumn{2}{|c|}{$\begin{array}{l}\text { Trab. de } \\
\text { conclusão } \\
\text { de curso }\end{array}$} & \multicolumn{2}{|c|}{ Livro } & \multicolumn{2}{|c|}{ Total } & \multicolumn{2}{|c|}{ Quinquênios } \\
\hline & $\mathrm{N}$ & $\%$ & $\mathrm{~N}$ & $\%$ & $\mathrm{~N}$ & $\%$ & $\mathrm{~N}$ & $\%$ & $\mathrm{~N}$ & $\%$ & $\mathrm{~N}$ & $\%$ & $\mathrm{~N}$ & $\%$ & $\mathrm{~N}$ & $\%$ & $\mathrm{~N}$ & $\%$ & $\mathrm{~N}$ & $\%$ & $\mathrm{~N}$ & $\%$ \\
\hline 1994 & 3 & 0,6 & 1 & 0,2 & 0 & 0 & 0 & 0 & 0 & 0 & 0 & 0 & 0 & 0 & 0 & 0 & 0 & 0 & 4 & 0,8 & & \\
\hline 1995 & 4 & 0,8 & 0 & 0 & 0 & 0 & 0 & 0 & 1 & 0,2 & 0 & 0 & 0 & 0 & 0 & 0 & 0 & 0 & 5 & 1 & & \\
\hline 1996 & 6 & 1,2 & 3 & 0,6 & 0 & 0 & 0 & 0 & 0 & 0 & 1 & 0,2 & 1 & 0,2 & 0 & 0 & 0 & 0 & 11 & 2,2 & & \\
\hline 1997 & 11 & 2,2 & 1 & 0,2 & 1 & 0,2 & 0 & 0 & 0 & 0 & 0 & 0 & 0 & 0 & 0 & 0 & 0 & 0 & 13 & 2,6 & & \\
\hline 1998 & 9 & 1,8 & 6 & 1,2 & 1 & 0,2 & 0 & 0 & 2 & 0,4 & 0 & 0 & 0 & 0 & 0 & 0 & 0 & 0 & 18 & 3,6 & 51 & 11,2 \\
\hline 1999 & 22 & 4,4 & 5 & 1 & 0 & 0 & 2 & 0,4 & 1 & 0,2 & 1 & 0,2 & 0 & 0 & 0 & 0 & 0 & 0 & 31 & 6,2 & & \\
\hline 2000 & 11 & 2,2 & 9 & 1,8 & 1 & 0,2 & 2 & 0,4 & 9 & 1,8 & 1 & 0,2 & 0 & 0 & 0 & 0 & 0 & 0 & 33 & 6,6 & & \\
\hline 2001 & 16 & 3,2 & 4 & 0,8 & 3 & 0,6 & 3 & 0,6 & 0 & 0 & 0 & 0 & 1 & 0,2 & 0 & 0 & 0 & 0 & 27 & 5,4 & & \\
\hline 2002 & 20 & 4 & 3 & 0,6 & 5 & 1 & 2 & 0,4 & 4 & 0,8 & 0 & 0 & 0 & 0 & 0 & 0 & 0 & 0 & 34 & 6,8 & & \\
\hline 2003 & 35 & 7 & 5 & 1 & 1 & 0,2 & 6 & 1,2 & 1 & 0,2 & 1 & 0,2 & 1 & 0,2 & 1 & 0,2 & 0 & 0 & 51 & 10,2 & 176 & 35,2 \\
\hline 2004 & 32 & 6,4 & 4 & 0,8 & 4 & 0,8 & 2 & 0,4 & 0 & 0 & 0 & 0 & 0 & 0 & 0 & 0 & 1 & 0,2 & 43 & 8,6 & & \\
\hline 2005 & 32 & 6,4 & 7 & 1,4 & 3 & 0,6 & 3 & 0,6 & 0 & 0 & 1 & 0,2 & 0 & 0 & 0 & 0 & 0 & 0 & 46 & 9,2 & & \\
\hline 2006 & 40 & 8 & 7 & 1,4 & 9 & 1,8 & 2 & 0,4 & 1 & 0,2 & 1 & 0,2 & 0 & 0 & 0 & 0 & 0 & 0 & 60 & 12 & & \\
\hline 2007 & 41 & 8,2 & 9 & 1,8 & 5 & 1 & 3 & 0,6 & 1 & 0,2 & 1 & 0,2 & 0 & 0 & 2 & 0,4 & 0 & 0 & 62 & 12,4 & & \\
\hline 2008 & 56 & 11,2 & 5 & 1 & 1 & 0,2 & 0 & 0 & 0 & 0 & 0 & 0 & 0 & 0 & 0 & 0 & 0 & 0 & 62 & 12,4 & 273 & 54,6 \\
\hline Total & 338 & 67,6 & 69 & 13,8 & 34 & 6,8 & 25 & 5 & 20 & 4 & 7 & 1,4 & 3 & 0,6 & 3 & 0,6 & 1 & 0,2 & 500 & 100 & 500 & 100 \\
\hline
\end{tabular}


Tabela 2. Categorias de estudos

\begin{tabular}{|c|c|c|c|c|c|c|c|c|c|c|c|c|}
\hline \multirow[t]{2}{*}{ Ano } & \multicolumn{2}{|c|}{ Avaliação } & \multicolumn{2}{|c|}{$\begin{array}{c}\text { Bibliográfico, teórico } \\
\text { ou documental }\end{array}$} & \multicolumn{2}{|c|}{$\begin{array}{l}\text { Avaliação do efeito de } \\
\text { programas }\end{array}$} & \multicolumn{2}{|c|}{$\begin{array}{l}\text { Descritivo de } \\
\text { intervenção }\end{array}$} & \multicolumn{2}{|c|}{$\begin{array}{c}\text { Mais de uma } \\
\text { categoria }\end{array}$} & \multicolumn{2}{|c|}{ Total } \\
\hline & $\mathrm{N}$ & $\%$ & $\mathrm{~N}$ & $\%$ & $\mathrm{~N}$ & $\%$ & $\mathrm{~N}$ & $\%$ & $\mathrm{~N}$ & $\%$ & $\mathrm{~N}$ & $\%$ \\
\hline 1994 & 3 & 0,6 & 0 & 0 & 1 & 0,2 & 0 & 0 & 0 & 0 & 4 & 0,8 \\
\hline 1995 & 3 & 0,6 & 1 & 0,2 & 0 & 0 & 1 & 0,2 & 0 & 0 & 5 & 1 \\
\hline 1996 & 10 & 2 & 0 & 0 & 0 & 0 & 1 & 0,2 & 0 & 0 & 11 & 2,2 \\
\hline 1997 & 12 & 2,4 & 0 & 0 & 0 & 0 & 1 & 0,2 & 0 & 0 & 13 & 2,6 \\
\hline 1998 & 10 & 2 & 4 & 0,8 & 1 & 0,2 & 3 & 0,6 & 0 & 0 & 18 & 3,6 \\
\hline 1999 & 19 & 3,8 & 6 & 1,2 & 3 & 0,6 & 3 & 0,6 & 0 & 0 & 31 & 6,2 \\
\hline 2000 & 21 & 4,2 & 5 & 1 & 3 & 0,6 & 1 & 0,2 & 3 & 0,6 & 33 & 6,6 \\
\hline 2001 & 21 & 4,2 & 4 & 0,8 & 0 & 0 & 0 & 0 & 2 & 0,4 & 27 & 5,4 \\
\hline 2002 & 22 & 4,4 & 4 & 0,8 & 3 & 0,6 & 5 & 1 & 0 & 0 & 34 & 6,8 \\
\hline 2003 & 34 & 6,8 & 11 & 2,2 & 3 & 0,6 & 3 & 0,6 & 0 & 0 & 51 & 10,2 \\
\hline 2004 & 36 & 7,2 & 1 & 0,2 & 2 & 0,4 & 3 & 0,6 & 1 & 0,2 & 43 & 8,6 \\
\hline 2005 & 35 & 7 & 4 & 0,8 & 4 & 0,8 & 2 & 0,4 & 1 & 0,2 & 46 & 9,2 \\
\hline 2006 & 42 & 8,4 & 7 & 1,4 & 8 & 1,6 & 1 & 0,2 & 2 & 0,4 & 60 & 12 \\
\hline 2007 & 43 & 8,6 & 5 & 1 & 7 & 1,4 & 5 & 1 & 2 & 0,4 & 62 & 12,4 \\
\hline 2008 & 42 & 8,4 & 2 & 0,4 & 11 & 2,2 & 2 & 0,4 & 5 & 1 & 62 & 12,4 \\
\hline Total & 353 & 70,6 & 54 & 10,8 & 46 & 9,2 & 31 & 6,2 & 16 & 3,2 & 500 & 100 \\
\hline
\end{tabular}

Tabela 3. Subcategorias por perspectiva de análise das avaliações

\begin{tabular}{|c|c|c|c|c|c|c|c|c|c|c|c|c|c|c|c|c|}
\hline \multirow[t]{2}{*}{ Ano } & \multicolumn{2}{|c|}{ Auto-referida } & \multicolumn{2}{|c|}{$\begin{array}{l}\text { Perceptivo- } \\
\text { auditiva }\end{array}$} & \multicolumn{2}{|c|}{$\begin{array}{c}\text { Ambiental e org. } \\
\text { do trab. }\end{array}$} & \multicolumn{2}{|c|}{ Comport. } & \multicolumn{2}{|c|}{ Acústica } & \multicolumn{2}{|c|}{ Médica } & \multicolumn{2}{|c|}{ Não especific. } & \multicolumn{2}{|c|}{ Total } \\
\hline & $\mathrm{N}$ & $\%$ & $\mathrm{~N}$ & $\%$ & $\mathrm{~N}$ & $\%$ & $\mathrm{~N}$ & $\%$ & $\mathrm{~N}$ & $\%$ & $\mathrm{~N}$ & $\%$ & $\mathrm{~N}$ & $\%$ & $\mathrm{~N}$ & $\%$ \\
\hline 1994 & 3 & 0,4 & 2 & 0,3 & 1 & 0,1 & 1 & 0,1 & 1 & 0,1 & 0 & 0 & 0 & 0 & 8 & 1,1 \\
\hline 1995 & 3 & 0,4 & 3 & 0,4 & 2 & 0,3 & 0 & 0 & 0 & 0 & 0 & 0 & 0 & 0 & 8 & 1,1 \\
\hline 1996 & 10 & 1,4 & 3 & 0,4 & 5 & 0,7 & 0 & 0 & 0 & 0 & 1 & 0,1 & 0 & 0 & 19 & 2,7 \\
\hline 1997 & 11 & 1,6 & 1 & 0,1 & 0 & 0 & 0 & 0 & 0 & 0 & 0 & 0 & 0 & 0 & 12 & 1,7 \\
\hline 1998 & 9 & 1,3 & 5 & 0,7 & 2 & 0,3 & 1 & 0,1 & 0 & 0 & 4 & 0,6 & 0 & 0 & 21 & 3 \\
\hline 1999 & 20 & 2,9 & 8 & 1,1 & 4 & 0,6 & 2 & 0,3 & 2 & 0,3 & 6 & 0,9 & 0 & 0 & 42 & 6 \\
\hline 2000 & 25 & 3,6 & 7 & 1 & 9 & 1,3 & 4 & 0,6 & 3 & 0,4 & 3 & 0,4 & 1 & 0,1 & 52 & 7,4 \\
\hline 2001 & 17 & 2,4 & 8 & 1,1 & 4 & 0,6 & 7 & 1 & 1 & 0,1 & 1 & 0,1 & 1 & 0,1 & 39 & 5,6 \\
\hline 2002 & 22 & 3,1 & 4 & 0,6 & 7 & 1 & 1 & 0,1 & 2 & 0,3 & 0 & 0 & 0 & 0 & 36 & 5,2 \\
\hline 2003 & 32 & 4,6 & 6 & 0,9 & 9 & 1,3 & 2 & 0,3 & 3 & 0,4 & 3 & 0,4 & 2 & 0,3 & 57 & 8,2 \\
\hline 2004 & 35 & 5 & 9 & 1,3 & 9 & 1,3 & 4 & 0,6 & 2 & 0,3 & 2 & 0,3 & 1 & 0,1 & 62 & 8,9 \\
\hline 2005 & 34 & 4,9 & 10 & 1,4 & 9 & 1,3 & 7 & 1 & 3 & 0,4 & 2 & 0,3 & 2 & 0,3 & 67 & 9,6 \\
\hline 2006 & 47 & 6,7 & 13 & 1,9 & 12 & 1,7 & 5 & 0,7 & 5 & 0,7 & 1 & 0,1 & 3 & 0,4 & 86 & 12,3 \\
\hline 2007 & 48 & 6,9 & 12 & 1,7 & 14 & 2 & 5 & 0,7 & 2 & 0,3 & 1 & 0,1 & 2 & 0,3 & 84 & 12 \\
\hline 2008 & 50 & 7,2 & 16 & 2,3 & 18 & 2,6 & 3 & 0,4 & 10 & 1,4 & 9 & 1,3 & 0 & 0 & 106 & 15,2 \\
\hline Total & 366 & 52,4 & 107 & 15,3 & 105 & 15 & 42 & 6 & 34 & 4,9 & 33 & 4,7 & 12 & 1,7 & 699 & 100 \\
\hline
\end{tabular}

Legenda: org. = organização; trab. = trabalho; comport. = comportamental; não especif. = não especificada

$=33 ; 1999=22$, totalizando 55 estudos) com subsequente progressão na quantidade dos estudos nos anos posteriores. Uma análise por quinquênio (Tabela 5) revela que o primeiro período (1994 a 1998) ofereceu apenas 10,2\% dos estudos (51), o segundo (1999 a 2003) acresceu em 35,2\% (176) e o número mais expressivo de contribuições corresponde ao último período (2004 a 2008), com 54,6\% das produções (273 títulos).

\section{DISCUSSÃO}

As pesquisas fonoaudiológicas com voz do professor são, na maioria $(338=67,6 \%)$, publicadas no formato de resumos em anais de eventos científicos, e em segundo lugar encontramse as publicações de artigos em periódicos $(69=13,8 \%)$, da mesma forma que foi constatado, na década de 1990, para 
Tabela 4. Estudos envolvendo análise vocal, por quinquênios

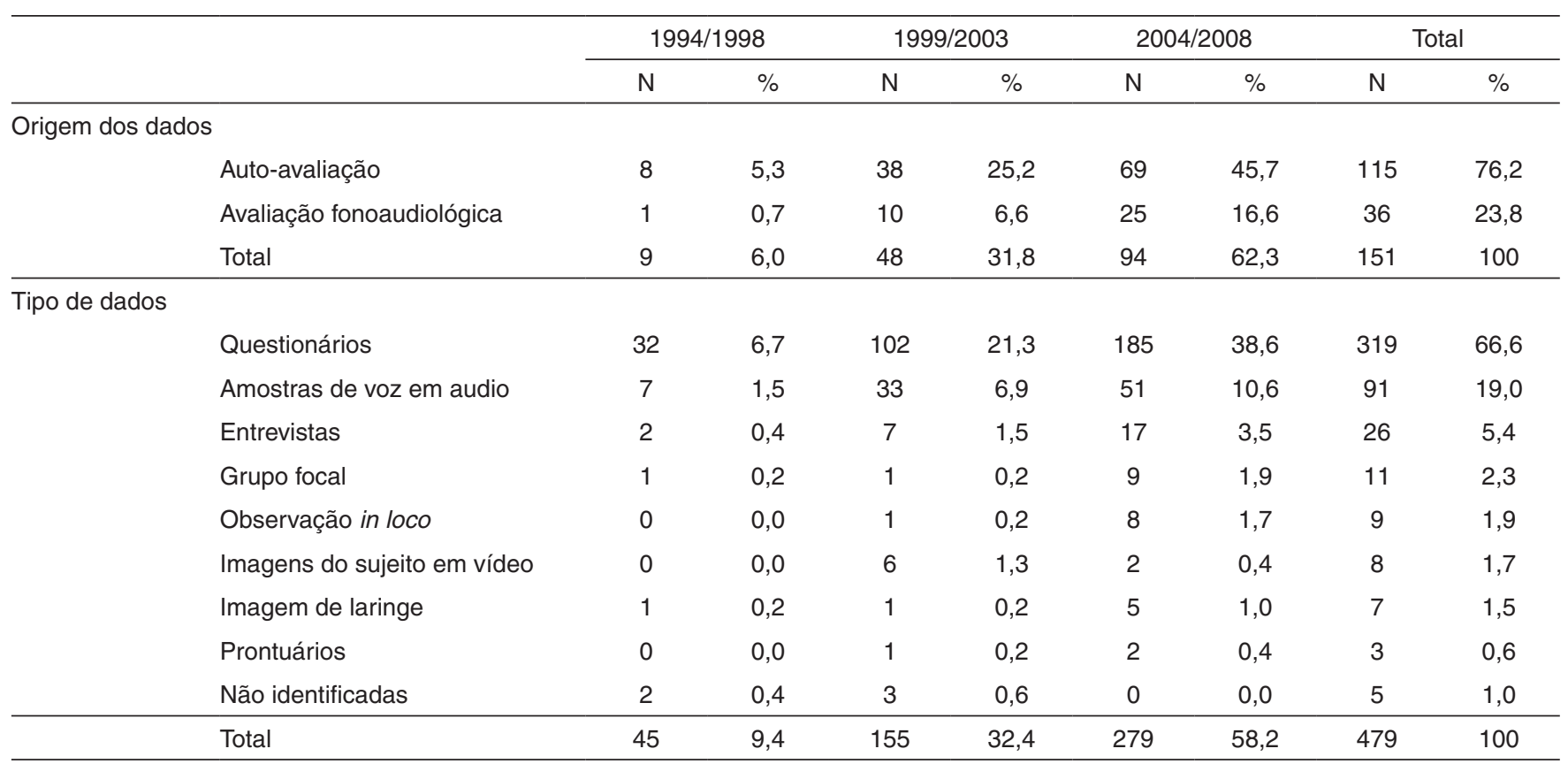

Tabela 5. Dados gerais analisados por quinquênios

\begin{tabular}{|c|c|c|c|c|c|c|c|}
\hline & \multicolumn{2}{|c|}{$1994 / 1998$} & \multicolumn{2}{|c|}{$1999 / 2003$} & \multicolumn{2}{|c|}{$2004 / 2008$} & \\
\hline & $\mathrm{N}$ & $\%$ & $\mathrm{~N}$ & $\%$ & $\mathrm{~N}$ & $\%$ & \\
\hline \multicolumn{8}{|l|}{ Tipo de publicação } \\
\hline Resumo em anais & 33 & 6,6 & 104 & 20,8 & 201 & 40,2 & \\
\hline Artigo & 11 & 2,2 & 26 & 5,2 & 32 & 6,4 & \\
\hline Capítulo de livro & 3 & 0,6 & 15 & 3,0 & 2 & 0,4 & \\
\hline Livro & 0 & 0,0 & 0 & 0,0 & 1 & 0,2 & \\
\hline Monografias de iniciação científica & 1 & 0,2 & 2 & 0,4 & 0 & 0,0 & \\
\hline Trabalho de conclusão de curso & 0 & 0,0 & 1 & 0,2 & 2 & 0,4 & \\
\hline Monografia de especialização & 0 & 0,0 & 15 & 3,0 & 10 & 2,0 & \\
\hline Tese ou dissertação de mestrado & 2 & 0,4 & 10 & 2,0 & 22 & 4,4 & \\
\hline Doutorado & 1 & 0,2 & 3 & 0,6 & 3 & 0,6 & \\
\hline Total & 51 & 10,2 & 176 & 35,2 & 273 & 54,6 & 500 \\
\hline \multicolumn{8}{|l|}{ Categorias de estudos } \\
\hline Avaliação & 38 & 7,6 & 117 & 23,4 & 199 & 39,8 & \\
\hline Avaliação do efeito de programas & 2 & 0,4 & 12 & 2,4 & 31 & 6,2 & \\
\hline Descritivo de intervenção & 6 & 1,2 & 12 & 2,4 & 13 & 2,6 & \\
\hline Bibliográfico, teórico ou documental & 5 & 1,0 & 30 & 6,0 & 19 & 3,8 & \\
\hline Mais de uma categoria & 0 & 0,0 & 5 & 1,0 & 11 & 2,2 & \\
\hline Total & 51 & 10,2 & 176 & 35,2 & 273 & 54,6 & 500 \\
\hline \multicolumn{8}{|l|}{ Subcategorias por perspectiva de análise } \\
\hline Acústica & 1 & 0,1 & 11 & 1,6 & 22 & 4,4 & \\
\hline Perceptivo-auditiva & 14 & 2,0 & 33 & 4,7 & 60 & 12 & \\
\hline Auto-referida & 36 & 5,2 & 116 & 16,6 & 214 & 42,8 & \\
\hline Médica & 5 & 0,7 & 13 & 1,9 & 15 & 3 & \\
\hline Comportamental & 2 & 0,3 & 16 & 2,3 & 24 & 4,8 & \\
\hline Ambiental e organizacional do trabalho & 10 & 1,4 & 33 & 4,7 & 62 & 12,4 & \\
\hline Não especificadas & 0 & 0,0 & 4 & 0,6 & 8 & 1,6 & \\
\hline Total & 68 & 9,7 & 226 & 32,3 & 405 & 81 & 699 \\
\hline
\end{tabular}


a divulgação de teses em saúde geral em anos anteriores ${ }^{(7)}$. Evidencia-se a realidade de que a maioria dos estudos não chega a ser publicados em periódicos, contentando-se os pesquisadores em divulgarem seu trabalho para seus pares em eventos com penetração limitada aos participantes. Entre os demais tipos de publicação encontram-se as teses de mestrado, as monografias de especialização, os capítulos de livro e as teses de doutorados, que perfazem juntas $17,2 \%$ da amostra (Tabela 1); e finalmente os tomos de iniciação científica, os trabalhos de conclusão de curso de graduação e um livro compõem os restantes as demais publicações sobre voz do professor deste levantamento.

Embora não tenha sido feita uma análise detalhada sobre as instituições de origem dos estudos, é evidente que os cursos de graduação e pós-graduação em Fonoaudiologia respondem pela maioria dessas publicações, uma realidade desde a década de 1980 para pesquisas na área da saúde ${ }^{(7)}$. Além disso, a inclusão de co-autores de outras formações acadêmicas não foi especificada, mas parece ser coincidente à existência de uma sociedade científica multiprofissional (a extinta Sociedade Brasileira de Laringologia e Voz - SBLV) ou de estudos desenvolvidos em Programas de Pós-Graduação em áreas diferentes da Fonoaudiologia.

A consideração mais abrangente sobre esta revisão de literatura é que a contribuição fonoaudiológica para o estudo da voz do professor foi consistente e crescente ao longo do período estudado, confirmando as percepções anteriores para áreas da saúde ${ }^{(7)}$ e especificamente na área de $v^{(8-11,19)}$, com $54,6 \%$ (da produção concentrada no último quinquênio analisado - 2004 a 2008 (Tabela 1).

Este crescimento é observado também nas publicações da área fonoaudiológica em geral $^{(4)}$, com a produção na área de voz quantitativamente em último lugar quando comparada a linguagem, audição e motricidade oral, fato preocupante para a consolidação do conhecimento na área. Os dados atuais reforçam a necessidade de incentivar publicações científicas em voz, levando-se em conta que a quantidade de publicações sobre voz do professor parece estabilizada e não crescente após 2006 (Tabela 1).

Alguns trabalhos apresentam baixa qualidade metodológica, com poucas análises estatísticas e, em alguns casos, conclusões errôneas, tal qual observado em revisão sistemática de publicações em voz e laringe em periódicos nacionais e internacionais ${ }^{(6)}$; no entanto, é perceptível que o desenho dos estudos vem se tornando mais detalhado e melhor controlado, o que mostra a intenção dos pesquisadores em produzir estudos que realmente tragam dados analisáveis.

A necessidade em se buscar uma distribuição mais equilibrada das temáticas estudadas é algo evidente e destacado em diversos estudos anteriores ${ }^{(4,5,16,17,19)}$. Nas publicações fonaudiológicas ${ }^{(4)}$ envolvendo áreas diversas, há maior predomínio de estudos que propõem avaliação e diagnóstico, fato também constatado entre os estudos específicos com voz do professor $^{(5,16,19)}$, o mesmo ocorre neste estudo, com 70,6\% na categoria avaliação (Tabela 2). Entre os estudos fonoaudiológicos em geral, a literatura aponta predomínio de pesquisas sobre o contexto clinico $^{(4)}$, o que se mostrou mais escasso $(3,2 \%)$ no caso das pesquisas com voz do professor (Tabela 2 ).
A pouca contribuição sobre promoção de saúde/prevenção de alterações vocais ${ }^{(5,19)}$ é corroborada pela baixa ocorrência de estudos descritivos de intervenção fonoaudiológica entre professores $(6,2 \%)$ e de avaliação do efeito de programas de saúde vocal $(9,2 \%)$, conforme pode-se ver na Tabela 2 .

A caracterização do problema vocal do professor é inicialmente feita por descrição dos sinais e sintomas e por análise perceptivo-auditiva das vozes dos professores. No que diz respeito ao tipo de sintomas referidos houve grande diversidade terminológica, com possibilidade somente de identificar algumas categorias de sintomas relacionados a alterações na qualidade vocal, com referências a cansaço e fadiga vocal, tensão e dores corporais proximais ou distais, e sensações desagradáveis durante a emissão vocal. Vale a pena destacar que, dos 151 estudos que envolvem referências a ocorrências de alterações vocais, 115 deles $(76,2 \%)$ analisam dados relatados pelo sujeito, ou seja, problemas auto-referidos, o que muitas vezes é confundido com a ocorrência real do problema (Tabela 4). Com a chegada dos programas de baixo custo para análise acústica e a implementação da estratégia de detalhamento visual da imagem laríngea nos estudos multiprofissionais, este panorama vem se modificando com aumento evidente de estudos com dados acústicos (22=64,7\% dos estudos com análise acústica) no período de 2004 a 2008 (Tabela 3).

A análise em relação à expressividade vocal teve um impulso mais recente, ampliando o olhar na direção dos aspectos de comunicação no processo ensino-aprendizagem, para a realidade da sala de aula, um avanço na busca de novas reflexões ${ }^{(1-3)}$. Da mesma forma, a necessidade de identificar as condições de uso da voz no trabalho de determinadas amostras populacionais dá origem a uma série de estudos descritivos, com deslocamento da ótica de análise da voz do sujeito para o ambiente e organização do trabalho docente $(105=15 \%$, Tabela 3). A temática, em destaque na literatura ${ }^{(16)}$, busca a identificação de problemas a serem minimizados em favor da saúde vocal dos professores.

Os protocolos de auto-avaliação, sobre diversos aspectos centrais ou relacionados ao problema de voz, têm trazido maior quantificação da perspectiva do sujeito sobre o seu problema. A utilização de protocolos padronizados nos estudos envolvendo auto-avaliação do professor foi citada em alguns resumos. Aparecem referências aos questionários de avaliação do impacto de um problema de voz na qualidade de vida como o Questionário de Qualidade de Vida em Voz (QVV), o Índice de Desvantagem Vocal (IDV) e o Protocolo de Participação e Atividades Vocais (PPAV); questionários para investigar aspectos do trabalho como JCQ (Job Content Questionnaire), CPV-P (Condição de Produção Vocal-Professor), TCBQ (Teacher Communication Behavior Questionnaire); e questionários sobre aspectos psíquicos e de estresse, como SRQ-20 (Self-Report Questionnaire), GHQ-12 (General Health Questionnaire), ISSL/ISS (Inventário de Sintomas Stress), e Trait Anxiety. Estudos para conhecer o perfil vocal ainda são os mais realizados, com uso predominante de questionários como instrumento de coleta de dados. Os inúmeros fatores de risco apontados pelos professores distribuem-se pelas seguintes categorias: alterações de saúde, fatores de personalidade dos professores, ambiente de trabalho e uso vocal. 
Como já apontado anteriormente ${ }^{(5,16)}$, poucos são os trabalhos em que foram realmente estudados os efeitos de intervenções fonoaudiológicas, mas, observa-se discreto movimento nesta direção, com maior número de trabalhos descritivos de intervenção no último quinquênio (13=42\% desta categoria - Tabela 5). Encontram-se presentes, também em maior quantidade neste período, descrições dos efeitos de programas de saúde vocal e de orientação vocal isolados ( $31=67 \%$ desta categoria - Tabela 5), sem apresentarem resultados consistentes que justifiquem sua prática.

Vários desses estudos são suportes para a elaboração de propostas de políticas públicas para o reconhecimento do distúrbio de voz como doença relacionada ao trabalho. O Centro de Referência em Saúde do Trabalhador de São Paulo (CEREST), preocupado com essa questão, em parceria com um grupo composto por diferentes técnicos e representantes dos profissionais que utilizam a voz como instrumento de trabalho, elaborou o documento Distúrbio de Voz Relacionado ao Trabalho ${ }^{(20)}$ (DRTV) com objetivo de auxiliar profissionais da voz em geral e, portanto, os professores, na prevenção, assistência, reabilitação, notificação e pagamento de benefícios. Destaca-se, então, a necessidade de uma produção científica cada vez mais eficaz no oferecimento de dados consolidados para embasar tais iniciativas.

É evidente, nesta análise dos 15 anos de produção em voz do professor, o papel de diversas instituições públicas e privadas que, ao autorizarem a realização de estudos com seus professores, demonstram interesse em contribuir com o progresso da ciência. Apesar desta colaboração, há uma grande distância entre favorecer a realização de um estudo e implementar mudanças sugeridas pelos seus resultados.
Da mesma forma, observa-se dificuldade em se implantar efetivamente leis na área de saúde vocal ${ }^{(21)}$, apesar das inúmeras pesquisas realizadas terem inspirado a proposta de diversas leis, em diferentes regiões do país, para prevenir alterações de voz, principalmente em professores. Estas, porém, quando aprovadas, encontram dificuldade no momento de sua implantação, ora pela falta de recurso, ora pela dificuldade em determinar os órgãos que irão gerenciá-las.

\section{COMENTÁRIOS FINAIS}

Apesar da preferência do fonoaudiólogo por estudar a voz do professor, constatada pelo grande número de produções científicas sobre o tema, a perspectiva da avaliação vocal tem sido privilegiada em relação à perspectiva relacionada aos aspectos específicos do trabalho docente, o que não impulsiona a atuação fonoaudiológica junto a este profissional da voz. Os estudos sobre efeitos de intervenções são mais recentes, descritivos, e, apesar de ainda pouco representativos, parecem indicar uma tendência de mudanças de foco que irá auxiliar a compreensão da complexa realidade do uso da voz na docência, nortear futuras ações fonoaudiológicas e até mesmo favorecer o reconhecimento do fonoaudiólogo como um prestador de serviço eficiente.

\section{AGRADECIMENTOS}

Aos fonoaudiólogos assistentes do CEV-SP cuja colaboração no levantamento bibliográfico foi fundamental para realização deste estudo.

\begin{abstract}
The purpose of this study was to review Brazilian publication regarding teachers' voice produced by Speech-Language Pathologists (SLPs) within the period from 1994 to 2008. This transversal study of bibliographic nature was carried out in three stages. The first consisted of data compilation from different sources; the second comprehended the search for the abstracts of the selected references; in the third stage, the publications were classified into four categories: individuals' assessment, treatment outcome evaluation, intervention description, and bibliographical/theoretical/documental researches. The final sample consisted of 500 publications, from which $415(83 \%)$ were related to the assessment categories. Among these, 357 (86\%) evaluated individuals and/or their working conditions, and 58 (14\%) had the aim to evaluate treatment outcome. Regarding analyses dimensions of the assessments, 202 studies (48.7\%) had more than one. Teachers' perspective (self-assessment) was the predominant dimension (52.5\% of the analyses), followed by auditory-perceptual analyses performed by SLPs (15.2\%), and evaluation of working environment and organization (14.9\%). The category intervention description had $31(6.2 \%)$ studies, and there were 54 (10.8\%) bibliographical/theoretical/documental researches. This literature review confirms that vocal assessment has been privileged in Brazilian researches regarding teachers' voice. Publications about treatment outcome evaluations are more recent and less representative, indicating a tendency of change of focus that might help the comprehension of the complex use of voice in teaching, and guide future studies on speech-language pathology intervention with these individuals.
\end{abstract}

Keywords: Voice; Faculty; Research; Review literature as topic; Voice disorders 


\section{REFERÊNCIAS}

1. Russo ICP, Ferreira LP. Fonoaudiólogos doutores no Brasil: análise das teses segundo áreas de atuação e programas. Pró-Fono. 2004;16(1):11930.

2. Campanatti-Ostiz H, Andrade CRF. Periódicos nacionais em Fonoaudiologia: caracterização estrutural. Rev Soc Bras Fonoaudiol. 2005;10(3): 147-54

3. Campanatti-Ostiz H, Andrade CRF. Periódicos nacionais em Fonoaudiologia: caracterização de indicador de impacto). Pró-Fono. 2006;18(1):99-110.

4. Berberian AP, Ferreira LP, Corteletti LCBJ, Azevedo JBM, Marques JM. A produção do conhecimento em Distúrbios da Comunicação: análise de periódicos (2000-2005). Rev Soc Bras Fonoaudiol. 2009;14(2):153-9.

5. Ferreira LP, Azevedo JBM. Análise de periódicos da Fonoaudiologia brasileiros: conhecer o passado para traçar o futuro na área de voz. Rev Fonoaudiol Universidad Valparaíso. 2008;2:43-8.

6. Vieira VP, De Biase N, Peccin MS, Atallah AN. The research questions and methological adequacy of clinical studies of the voice and larynx published in Brazilian and international journals. J Eval Clin Pract. 2009;15(3):473-7.

7. Nunes ED. A review of research studies conducted on scientific production in collective health in Brazil. Scientometrics, 1999;44(2):157-67.

8. Simões M. A voz do professor: histórico da produção científica de fonoaudiólogos brasileiros sobre o uso da voz nessa categoria profissional. In: Ferreira LP, Oliveira SMRP, organizador. Voz profissional: produção científica da fonoaudiologia brasileira. São Paulo: Roca; 2004. p. 1-9.

9. Simões M. A voz do professor: histórico da produção científica de fonoaudiólogos brasileiros sobre o uso da voz nesta categoria profissional. In: Ferreira LP, Oliveira, SMR, organizador. Voz profissional II: produção científica da fonoaudiologia brasileira [CD-ROM]. São Paulo: Comitê de Voz da Sociedade Brasileira de Fonoaudiologia; janeiro/2004 a julho/2005.

10. Dragone MLS, Ferreira LP, Simões-Zenari M, Giannini SP. In: Oliveira IB, Almeida AAF, Raize T, organizador. A voz do professor. Voz profissional: produção científica da fonoaudiologia brasileira. Revisão Ampliada (2005-2007). Rev Soc Bras Fonoaudiol [Internet]. Suplemento 2008. [citado 2010 Jan 4]. Disponível em: http://www.sbfa.org.br/portal/ pg.php?id=depto_motricidade\&ttpg_comissao $=V O Z \&$ tpc $=$ cinza\&ttpg $/$ 11. Oliveira IB, Almeida AAF, Raize T, organizador. A voz do professor.
Voz profissional: produção científica da fonoaudiologia brasileira. Revisão Ampliada (2005-2007). Rev Soc Bras Fonoaudiol [Internet]. Suplemento 2008. [citado 2010 Jan 4]. Disponível em: http://www.sbfa. org.br/portal/voz_profissional/index.htm

12. Pinto AMM, Furk MAE. Projeto saúde vocal do professor. In: Ferreira LP, organizador. Trabalhando a voz. São Paulo: Summus; 1987. p. 1127.

13. Verdolini K, Ramig LO. Review: occupational risks for voice problems. Logoped Phoniatr Vocol. 2001;26(1):37-46.

14. Roy N, Merrill RM, Thibeault S, Gray SD, Smith EM. Voice disorders in teachers and the general population: effects on work performance, attendance, and future career choices. J Speech Lang Hear Res. 2004;47(3):542-51.

15. Behlau M, Zambon F, Guerrieri AC, Roy N. Panorama epidemiológico sobre a voz do professor no Brasil. Anais do $17^{\circ}$ Congresso Brasileiro de Fonoaudiologia. $1^{\circ}$ Congresso Íbero-Americano de Fonoaudiologia. Rev Soc Bras Fonoaudiol [Internet]. Suplemento 2009: 1511. [citado 2009 Dez 1].Disponível em:http://www.sbfa.org.br/portal/anais2009/ anais_select.php?op=PR\&cid $=1511 \&$ tid $=1$

16. Leite MP, Souza AN. Condições de trabalho e suas repercussões na saúde dos profesores da educação básica no Brasil: estado da arte [Internet]. Campinas: Fundacentro; 2007. [citado 2010 Abr 5]. Disponível em http://www.fundacentro.gov.br/dominios/CTN/anexos/ relatorio_unicamp_corrigido.pdf

17. Romano CC, Alves LA, Silva LA, Marziale MHP, Robazzi MLCC. Alterações vocais decorrentes do trabalho em professores: uma revisão de literatura. Rev Enferm UFPE. 2009;3(3): 269-77.

18. Reed CG. Voice therapy: a need for research. J Speech Hear Disord. 1980;45(2):157-69.

19. Dragone MLS, Behlau M. A fonoaudiologia brasileira e a voz do professor: olhares científicos no decorrer do tempo. Rev Fonoaudiol Bras. 2006;4(2):6-9.

20. Secretaria de Estado de Saúde de São Paulo. Centro de Referência de Saúde do Trabalhador. CEREST-SP. Distúrbios da voz relacionados ao trabalho. Bol Epidemiol Paul [Internet]. 2006 [citado 2009 Dez 29]; 3(26):16-22. Disponível em: http://www.cve.saude.sp.gov.br/agencia/ bepa26_dist.htm

21. Ferreira LP, Servilha EAM, Masson MLV, Reinaldi MBM. Políticas públicas e voz do professor: caracterização das leis brasileiras. Rev Soc Bras Fonoaudiol. 2009;14(1):1-7. 